\title{
Relative Motion of Formation Flying with Elliptical Reference Orbit
}

\author{
Hany R Dwidar ${ }^{\dagger}$ and Ashraf H. Owis ${ }^{\dagger}$ \\ ${ }^{\dagger}$ Department of Astronomy, Space and Meteorology, Faculty of Science, Cairo University
}

\begin{abstract}
In this paper we present the optimal control of the relative motion of formation flying consisting of two spacecrafts. One of the spacecraft is considered as the chief, orbiting the Earth on a Highly Elliptical Orbit(HEO), and the other ,orbiting the chief, is considered as the deputy. The Keplerian relative dyanmics of the formation as well as the the second zonal hamonics of the Earth's gravitational field $\left(J_{2}\right)$ are studied. To study these perturbative effect the linearized true anomaly varying Tschauner-Hempel equations are augmented to include the effect of $\mathbf{J}_{2}$. We solve the nonlinear feedback optimal control of the relative motion using the state dependent Riccati Equation(SDRE). The results are validated through a nummerical example.
\end{abstract}

\section{INTRODUCTION}

The multi-spacecraft mission have proved powerful than the monolithic ones in the sense of reliability Reconfigurability, and redundancy. In addition to large apertures in the interferometric missions and therefore longer baseline. The new challenging technology require a high- Precision relative orbit control. Relative motion between a chief and a chaser spacecraft has been extensively studied over past several decades. The well-known Clohesssy-Wiltshire(CW) equations[1] originally known as Hills equations[2] used to study the linearised equation of motion around the orbit of the chief satellite, which is circular and subject to the Keplerian motion only. Other models have been introduced in which the chief orbit is eccentric subject to the non-Keplerian perturbation forces [3], [4], [5], [6], [7], [8] .For near Earth space missions, the second zonal harmonic $\left(J_{2}\right)$ perturbation is the dominant in for long term modelling context, and therefore has drawn considerable attention [9], [10], [11]. An analytic solution introduced [4]. A numerical solution based on the linear quadratic regulator (LQR), with limited thrust implemented, has been developed in [12]. The feedback optimal control of the relative motion of sun-facing formation flying using the generating function technique introduced by Scheeres 2006 to solve the Linear True Anomaly Variant Quadratic Regulator(LTAVQR) has been developed [16]. One of the most common strategies of controlling the relative position of a formation of satellite, is the chief and deputy strategy. In which, one of the spacecraft is considered as the chief, orbiting the Earth on a Highly Elliptical Orbit(HEO), and the other ,orbiting the chief, is considered as the deputy. The reference orbit of the chief spacecraft is elliptic and the Tschauner-Hempel equations are used to formulated the dynamical model based on the gravitational filed of the Earth up to the second zonal harmonics. We get closed loop feedback optimal control solution based on the State Dependent Riccati Equation(SDRE) that is able to accommodate some errors in the initial condition.

\section{STAtMent OF THE PROBlem}

Due to the limitation of the Cartesian coordinate system, we use the Local Vertical Local Horizontal (LVLH)coordinate system to overcome some drawbacks incurred by the Cartisan one such as, equation linearization and perturbation inclusion. We study the motion of two-spacecraft formation flying moving under the main gravitational field of the Earth and the second zonal harmonic. The chief spacecraft will move on an elliptic orbit described by the orbital elements $(a, e, i, \Omega, \omega, \theta)$ as shown in Figure 1 and the chaser one will be described with the chief's orbit as reference. The equation of motion can be written as[13], [14], [15]

$$
\ddot{\vec{r}}=\vec{g}(\vec{r})+\vec{J}(\vec{r})
$$

where $\vec{g}$, and $\vec{J}$ are accelerations due to the spherical and oblate Earth.

We assume that the chief spacecraft is at reference orbit $\vec{R}_{f c}$ and the chaser spacecraft at position vector $\vec{R}$. We can use equ(1) to write the accelerations of the two spacecrafts

$$
\begin{aligned}
\ddot{\vec{R}} & =\vec{g}(\vec{R})+\vec{J}(\vec{R})+\vec{a}(\vec{R}) \\
\ddot{\overrightarrow{\vec{R}}} & =\vec{g}\left(\overrightarrow{R_{f c}}\right)+\vec{J}\left(\overrightarrow{R_{f c}}\right)+\vec{a}\left(\overrightarrow{R_{f c}}\right)
\end{aligned}
$$

where we have $\left(\vec{R}_{f c}\right)$ and $(\vec{R})$ are defined as follows

$$
\begin{array}{rlrl}
\vec{R}_{f c} & =R_{f c} \hat{i} & & \text { (Non-inertial frame) } \\
\vec{R} & =\left(R_{f c}+x\right) \hat{i}+y \hat{j}+z \hat{k} & \text { (Non-inertial frame) }
\end{array}
$$

\section{A. Equation of motion of the relative motion}

To find the relative acceleration in the inertial frame $i \ddot{\vec{\rho}}$ (the derivative in the inertial frame is identified by $i$ ) we compute

$$
\ddot{\vec{R}}-\vec{R}_{f c}=\vec{g}(\vec{R})-\vec{g}\left(\vec{R}_{f c}\right)+\vec{J}(\vec{R})-\vec{J}\left(\vec{R}_{f c}\right)+\vec{a}(\vec{R})-\vec{a}\left(\vec{R}_{f c}\right)
$$


The relative acceleration in the non-inertial frame $\ddot{\vec{\rho}}=\left[\begin{array}{c}\ddot{x} \\ \ddot{y} \\ \ddot{z}\end{array}\right]$ is given by

$$
\ddot{\vec{\rho}}=i \ddot{\vec{\rho}}-2 \dot{\vec{\theta}} \times \dot{\vec{\rho}}-\dot{\vec{\theta}} \times(\dot{\vec{\theta}} \times \vec{\rho})-\ddot{\vec{\theta}} \times \vec{\rho}
$$

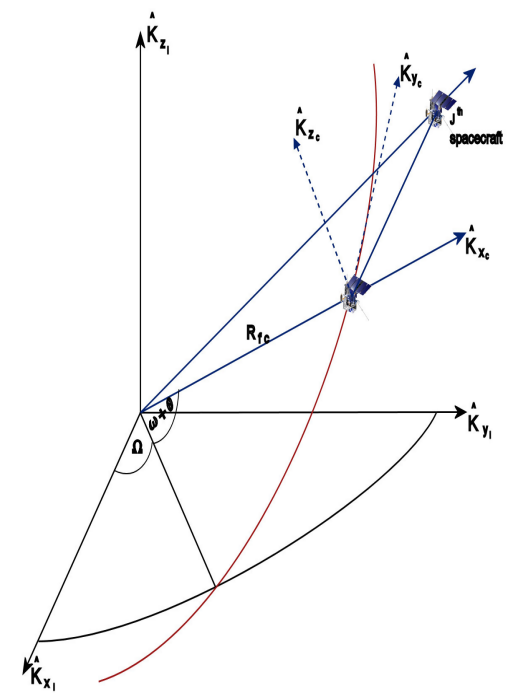

Figure 1.

Where $\dot{\vec{\theta}}, \ddot{\vec{\theta}}$ correspond to the angular velocity and acceleration of this orbiting reference frame.

$$
\begin{aligned}
\dot{\vec{\theta}} & =\dot{\theta} \hat{k} \\
\left|\vec{R}_{f c}\right| & =\frac{a\left(1-e^{2}\right)}{1+e \cos f} \\
\dot{\theta} & =\frac{n(1+e \cos \theta)^{2}}{\left(1-e^{2}\right)^{3 / 2}}
\end{aligned}
$$

The relation between time and true anomaly is given by

$$
t-t_{p}=\frac{1}{n}\left[2 \arctan \left(\sqrt{\frac{1-e}{1+e}} \tan \frac{\theta}{2}\right)-\frac{e \sqrt{1-e^{2}} \sin \theta}{1+e \cos \theta}\right]
$$

Where $n=\left(\mu / a^{3}\right)^{1 / 2}$ is the mean motion of the reference orbit.

If we use $\theta$ as the free variable, the equation of motion can be transformed using the relationships $(\cdot)^{\bullet}=(\cdot)^{\prime} \dot{\theta}, \quad(\cdot)^{\bullet \bullet}=(\cdot)^{\prime \prime} \dot{\theta}^{2}+\dot{\theta} \dot{\theta}^{\prime}(\cdot)^{\prime}$

Using the above equation we get

$$
\frac{d}{d t}\left[\begin{array}{c}
\dot{x} \\
\dot{y} \\
\dot{z}
\end{array}\right]=\left[\begin{array}{c}
x^{\prime \prime} \dot{\theta}^{2}+\dot{\theta} \dot{\theta}^{\prime} x^{\prime} \\
y^{\prime \prime} \dot{\theta}^{2}+\dot{\theta} \dot{\theta}^{\prime} y^{\prime} \\
z^{\prime \prime} \dot{\theta}^{2}+\dot{\theta} \dot{\theta}^{\prime} z^{\prime}
\end{array}\right]
$$

From equ (4) we can write the state equation of system in terms of $(\theta)$ as the free variables as follows

$$
\frac{d}{d \theta}\left[\begin{array}{c}
x \\
y \\
z \\
x^{\prime} \\
y^{\prime} \\
z^{\prime}
\end{array}\right]=\left[\begin{array}{c}
x^{\prime} \\
y^{\prime} \\
z^{\prime} \\
\frac{\ddot{x}-\dot{\theta} \dot{\theta}^{\prime} x^{\prime}}{\dot{\theta}^{2}} \\
\frac{\ddot{y}-\dot{\theta}^{\prime} \dot{\theta}^{\prime}}{\dot{\theta}^{2}} \\
\frac{\ddot{z}-\dot{\theta}^{\prime} z^{\prime}}{\dot{\theta}^{2}}
\end{array}\right]
$$

Where $\dot{\theta}^{\prime}=\frac{-2 n(1+e \cos \theta) e \sin \theta}{\left(1-e^{2}\right)^{3 / 2}}$ 


\section{B. The gravitational acceleration}

To find the garvitional acceleration due to the spherical Earth and the second zonal harmonics $\left(J_{2}\right)$ term we should write the gravitational potential in the following augmented form

$$
U(r)=\frac{\mu}{r}-\frac{3 \mu J_{2} R_{\oplus}^{2} z^{2}}{2 r^{5}}+\frac{\mu J_{2} R_{\oplus}^{2}}{2 r^{3}}
$$

and hence the acceleration resulting from this potential will be

$$
\begin{aligned}
\vec{r} & =-\frac{\mu}{r^{3}} \vec{r}+\frac{15 \mu J_{2} R_{\oplus}^{2} z^{2}}{2 r^{6}} \vec{r}-\frac{3 \mu J_{2} R_{\oplus}^{2}}{2 r^{4}} \vec{r}-\frac{3 \mu J_{2} R_{\oplus}^{2} z^{2}}{r^{5}} \hat{K} \\
& = \\
g & \vec{r})+\vec{J}(\vec{r})
\end{aligned}
$$

where $\vec{g}$ and $\vec{J}$ are the the accelerations of the spherical and oblate Earth reseptively and $\hat{K}$ is unit vector in the inertial ECI frame.

The last term can be written in the orbiting non-inertial frame as follows

$$
-\frac{3 \mu J_{2} R_{\oplus}^{2}}{r^{4}}\left(\begin{array}{c}
\sin (\Omega) \sin ^{2}(i) \\
-\cos (\Omega) \sin ^{2}(i) \\
\cos (i) \sin (i)
\end{array}\right)
$$

Within the assumption that $|\vec{\rho}|<<\left|\vec{R}_{f c}\right|$ We can write

$$
\vec{g}(\vec{R})-\vec{g}\left(\overrightarrow{R_{f c}}\right)=\frac{-\mu}{\left|\vec{R}_{f c}\right|^{3}}(-2 x \hat{i}+y \hat{j}+z \hat{k})
$$

Also we have

$$
\vec{J}(\vec{R})-\vec{J}\left(\vec{R}_{f c}\right)=6 \frac{\mu J_{2} R_{\oplus}^{2}}{R_{f c}^{5}} A(\theta)\left[\begin{array}{l}
x \\
y \\
z
\end{array}\right]
$$

where $A(\theta)=$

$$
\left[\begin{array}{ccc}
1-3(\sin i \sin (\theta+\omega))^{2} & \sin 2(\theta+\omega) \sin ^{2} i & \sin 2 i \sin (\theta+\omega) \\
\sin 2(\theta+\omega) \sin ^{2} i & -\frac{1}{4}-\frac{1}{2} \sin ^{2} i+\frac{7}{4}(\sin i \sin (\theta+\omega))^{2} & -\frac{1}{4} \sin 2 i \cos (\theta+\omega) \\
\sin 2 i \sin (\theta+\omega) & -\frac{1}{4} \sin 2 i \cos (\theta+\omega) & -\frac{3}{4}+\frac{1}{2} \sin ^{2} i+\frac{5}{4}(\sin i \sin (\theta+\omega))^{2}
\end{array}\right]
$$

Pluging equs 9, and 10 into equ. 3 and then substituting into equ.5 we get the state equation of the system as

$$
X^{\prime}=A(\theta) X+B(\theta) U
$$

where $\quad X=\left[x, y, z, x^{\prime}, y^{\prime}, z^{\prime}\right]$ is the state vector and $U=\left[u_{x}, u_{y}, u_{z}\right]$ is the control vector

$$
\begin{gathered}
A(\theta)=\left[\begin{array}{llllll}
a_{11} & a_{12} & a_{13} & a_{14} & a_{15} & a_{16} \\
a_{21} & a_{22} & a_{23} & a_{24} & a_{25} & a_{26} \\
a_{31} & a_{32} & a_{33} & a_{34} & a_{35} & a_{36} \\
a_{41} & a_{42} & a_{43} & a_{44} & a_{45} & a_{46} \\
a_{51} & a_{52} & a_{53} & a_{54} & a_{55} & a_{56} \\
a_{61} & a_{62} & a_{63} & a_{64} & a_{65} & a_{66}
\end{array}\right] \\
B(\theta)=\frac{\left(1-e^{2}\right)^{3}}{(1+e \cos \theta)^{4} n^{2}}\left[\begin{array}{ccc}
0 & 0 & 0 \\
0 & 0 & 0 \\
0 & 0 & 0 \\
1 & 0 & 0 \\
0 & 1 & 0 \\
0 & 0 & 1
\end{array}\right]
\end{gathered}
$$

where 


$$
\begin{aligned}
& a_{14}=1, \quad a_{11}=a_{12}=a_{13}=a_{15}=a_{16}=0, \\
& a_{21}=a_{22}=a_{23}=a_{24}=a_{26}=0, \quad a_{25}=1 \text {, } \\
& a_{34}=a_{31}=a_{35}=a_{32}=a_{33}=0, \quad a_{36}=1, \\
& a_{41}=\frac{3+e \sin \theta}{1+e \cos \theta}+\frac{6 J_{2} R_{\oplus}^{2}}{a^{2}\left(1-e^{2}\right)^{2}}\left(1-3(\sin i \sin (\theta+\omega))^{2}\right)(1+e \cos \theta), \\
& a_{42}=-\frac{2 e \sin \theta}{1+e \cos \theta}+\frac{6 J_{2} R_{\oplus}^{2}}{a^{2}\left(1-e^{2}\right)^{2}}\left(\sin 2(\theta+\omega) \sin ^{2} i\right)(1+e \cos \theta), \\
& a_{43}=\frac{6 J_{2} R_{\oplus}^{2}}{a^{2}\left(1-e^{2}\right)^{2}} \sin 2 i \sin ((\theta+\omega)(1+e \cos \theta), \\
& a_{44}=\frac{2 e \sin \theta}{1+e \cos \theta}, \\
& a_{45}=2 \text {, } \\
& a_{46}=0 \text {, } \\
& a_{51}=\frac{2 e \sin \theta}{1+e \cos \theta}+\frac{6 J_{2} R_{\oplus}^{2}}{a^{2}\left(1-e^{2}\right)^{2}} \sin 2(\theta+\omega) \sin ^{2} i(1+e \cos \theta), \\
& a_{52}=\frac{e \cos \theta}{1+e \cos \theta}+\frac{3 J_{2} R_{\oplus}^{2}}{a^{2}\left(1-e^{2}\right)^{2}}\left(-\frac{1}{2}-\sin ^{2} i+\frac{7}{2}(\sin i \sin (\theta+\omega))^{2}\right)(1+e \cos \theta), \\
& a_{53}=-\frac{3 J_{2} R_{\oplus}^{2}}{2 a^{2}\left(1-e^{2}\right)^{2}}(\sin 2 i \cos ((\theta+\omega))(1+e \cos \theta), \\
& a_{54}=-2 \text {, } \\
& a_{55}=\frac{2 e \sin \theta}{1+e \cos \theta}, \\
& a_{56}=0 \text {, } \\
& a_{64}=a_{65}=0, \\
& a_{61}=\frac{6 J_{2} R_{\oplus}^{2}}{a^{2}\left(1-e^{2}\right)^{2}} \sin 2 i \sin ((\theta+\omega)(1+e \cos \theta), \\
& a_{62}=-\frac{3 J_{2} R_{\oplus}^{2}}{2 a^{2}\left(1-e^{2}\right)^{2}} \sin 2 i \cos (\theta+\omega)(1+e \cos \theta), \\
& a_{63}=\frac{-1}{1+e \cos \theta} \\
& +\frac{3 J_{2} R_{\oplus}^{2}}{2 a^{2}\left(1-e^{2}\right)^{2}}\left(-3+2 \sin ^{2} i+5(\sin i \sin (\theta+\omega))^{2}\right)(1+e \cos \theta), \\
& a_{66}=\frac{2 e \sin \theta}{1+e \cos \theta} \text {, }
\end{aligned}
$$

\section{State Dependent Riccati EQuation}

Consider the consider the State Dependent Linear Quadratic Regulator written as follows:

$$
\dot{\mathbf{x}}=\mathbf{A}(\mathbf{x}) \mathbf{x}(t)+\mathbf{B}(\mathbf{x}) \mathbf{u}(t), \quad \mathbf{x}\left(t_{0}\right)=\mathbf{x}_{\mathbf{0}} \in \mathbb{R}^{n}
$$

where $\mathbf{x}(t) \in \mathbb{R}^{n}$ is the state vector and $\mathbf{u}(t) \in \mathbb{R}^{m}$ is the control vector.

The optimization problem is to find the control $\mathbf{u}^{*}$ that minimizes the cost function :

$$
J_{L Q R}=\frac{1}{2} \int_{t_{0}}^{t_{f}}\left(\mathbf{x}^{T} \mathbf{Q} \mathbf{x}+\mathbf{u}^{T} \mathbf{R} \mathbf{u}\right) d t
$$

where $\mathbf{Q}$ and $\mathbf{R}$ are the weight matrices.

State Dependent Riccati Equation The feedback optimal solution of the above problem $\mathbf{u}^{*}$ is given by 


$$
\mathbf{u}^{*}(\mathbf{x})=-\mathbf{R}^{-1}(\mathbf{x}) \mathbf{B}^{T}(\mathbf{x}) \mathbf{P}(\mathbf{x}) \mathbf{x}
$$

Where $\mathbf{P}(\mathbf{x})$ is obtained by solving the SDRE State Dependent Riccati equation:

$$
\begin{array}{r}
\dot{\mathbf{P}}(\mathbf{x})+\mathbf{A}^{T}(\mathbf{x}) \mathbf{P}(\mathbf{x})+\mathbf{P}(\mathbf{x}) \mathbf{A}(\mathbf{x})+\mathbf{Q}(\mathbf{x})- \\
\mathbf{P}(\mathbf{x}) \mathbf{B}(\mathbf{x}) \mathbf{R}^{-1}(\mathbf{x}) \mathbf{B}^{T}(\mathbf{x}) \mathbf{P}^{T}(\mathbf{x})=0
\end{array}
$$

We note that the Riccati matrix, $\mathbf{P}(\mathbf{x})$ depends on the choice of $\mathbf{A}(\mathbf{x})$, and since $\mathbf{A}(\mathbf{x})$ is not unique we have multiple optimal solutions.

\section{Factored Controllability}

For the factored system equ.(11) the controllability is established by verifying that the controllability matrix

$$
\mathbf{M}_{c l}=\left[\mathbf{B} \mathbf{A B} \mathbf{A}^{2} \mathbf{B} \mathbf{A}^{3} \mathbf{B}\right]
$$

has a rank equals to $n=6 \forall x$ in the domain.

Since $\mathbf{A}$ and $\mathbf{B}$ have nonvanishing rows the controllability matrix $\mathbf{M}_{c l}$ for the System equ.(11) is of rank 6 .

\section{NumERICAL EXAMPLE}

The elements of the reference satellite are

$\begin{array}{lll}\text { eccentricity } & = & 0.6 \\ \text { Semi-major axis } & = & 60 * 10^{6} \mathrm{~m} \\ \text { inclination } & = & \mathrm{PI} / 3.0 \mathrm{rad} \\ \text { argument of perigee } & = & 0 \mathrm{rad} \\ \text { right ascention of the ascending node } & = & 0.69813 \mathrm{rad}\end{array}$

and the intial condition are

$$
\left.\begin{array}{lll}
\theta_{0} & =-0.1 \mathrm{rad} \\
X_{0} & = & {[150,1,1,0,0}
\end{array}\right]
$$

and the final condition are

$$
\begin{aligned}
& \begin{array}{l}
\theta_{f}=0.1 \mathrm{rad} \\
X_{f}=[150,1,1,0,00]
\end{array} \\
& Q(t)=\left[\begin{array}{llllll}
1 & 0 & 0 & 0 & 0 & 0 \\
0 & 1 & 0 & 0 & 0 & 0 \\
0 & 0 & 1 & 0 & 0 & 0 \\
0 & 0 & 0 & 1 & 0 & 0 \\
0 & 0 & 0 & 0 & 1 & 0 \\
0 & 0 & 0 & 0 & 0 & 1
\end{array}\right] \\
& Q_{f}(t)=\left[\begin{array}{llllll}
0 & 0 & 0 & 0 & 0 & 0 \\
0 & 0 & 0 & 0 & 0 & 0 \\
0 & 0 & 0 & 0 & 0 & 0 \\
0 & 0 & 0 & 0 & 0 & 0 \\
0 & 0 & 0 & 0 & 0 & 0 \\
0 & 0 & 0 & 0 & 0 & 0
\end{array}\right]
\end{aligned}
$$




\section{CONCLUSIONS}

- The feedback optimal control of relative motion of formation flying problem is solved by linearizing the original nonlinear dyanmics.

- The time varying linearized problem has been solved using the State Dependent Riccati Equation technique.

- The method can be used for arbitrary boundary condition.

- The result is valid for any short time span formation flying rendezvous maneuver.

ACKNOWLEDGEMENTS

This project was supported financially by the Science and Technology Development Fund (STDF), Egypt, Grant No 666

\section{REFERENCES}

[1] Clohessy, W.H., Wiltshire, R.S., Terminal Guidance System for Satellite Rendezvous, Journal of the Aerospace Sciences, Vol. 27, No. 9, 1960, pp. 653-658.

[2] Hill, G., Researches in Lunar Theory, American Journal of Mathematics, Vol. 1, 1878, pp.5-26

[3] Wiesel, W.E., Relative Satellite Motion About an Oblate Planet, Journal of Guidance, Control, and Dynamics, Vol. 25, No. 4, 2002, pp. 776-785.

[4] Melton, R., Time-Explicit Representation of Relative Motion Between Elliptical Orbits, Journal of Guidance, Control, and Dynamics, Vol. 23, No. 4, 2000, pp. 604-610.

[5] Tschauner, J., Hempel, P., Rendezvous Zu Einem In Elliptischer Bahn Umlaufenden Ziel, Astronautica Acta, Vol. 11, No. 2, 1965, pp. 104-109

[6] Inalhan, G. ,Tillerson, M. and How, J. P. Relative Dynamics and Control of Spacecraft Formation in Eccentric Orbits, Journal of Guidance Control and Dynamics Vol. 25, No. 61, PP. 48-59

[7] Gurfil, P., Relative Motion Between Elliptic Orbits Generalized Boundedness Conditions and Optimal Formation keeping, Journal of Guidance, Control, and Dynamic Vol. 28, No. 4, 2005, pp. $761-767$

[8] Palmer, P.L., Imre, E., Relative Motion Between Satellites on Neighboring Keplerian Orbits, Journal of Guidance, Control, and Dynamics, Vol. 30, No. 2,2007 , pp. 521-528.

[9] Schweighart, S. A., Sedwick, and R. J., High-Fidelity Linearized $J_{2}$ Model for Satellite Formation Flight, Journal of Guidance Control and Dynamics Vol. 25, No. 6, PP. 1173-1180

[10] Schaub, H., and Alfriend, K. T., J, J2 Invariant Orbits for Spacecraft Formations, Celestial Mechanics and Dynamical Astronomy, Vol. 79, 2001, pp. $77-95$.

[11] Vadali, S.R, Sengupta, P., Yan, H., Alfriend, K.T., Fundamental Frequencies of Satellite Relative Motion and Control of Formations, Journal of Guidance, Control, and Dynamics, Vol. 31, No. 5, 2008, pp. 1239-1248

[12] Lantoine, G., Epenoy, R.,A Quadratically-Constrained Linear Quadratic Regulator Approach for Finite-Thrust Orbital Rendezvous, Journal of Guidance, Control and Dynamics, Vol. 35, No. 6, 2012, pp. 1787-1797

[13] Capo-Lugo, P.A., and, Banium, P.M., Active control Schemes to Satisfy Separation Distance Constraints, Journal of Guidance Control and Dynamics Vol. 30, No. 4, PP. 1152-1155

[14] Theron, A., Jouhaud, F. and Chretien, J.-P., Modelisation du Mouvement Orbital Relatif entre Deux Satellites, Note interne 1/08282, ONERA, January 2004.

[15] Perea, L., D'AmicoS., and Elosegui, P., Relative Orbit Control of a Virtual Telescope in an Eccentric Orbit, 21st International Symposium on Space Flight Dynamics, 28 Sep. -2 Oct. 2009, Toulouse, France (2009).

[16] Owis, A. and Dwidar, H. , Feedback Optimal Control of Relative Motion for Elliptical Reference Orbit of Sun-Facing Formation Flying, New Trends in Astrodynamics and Applications VI New York University, New York City June 6-8, 2011 




Figure 2.

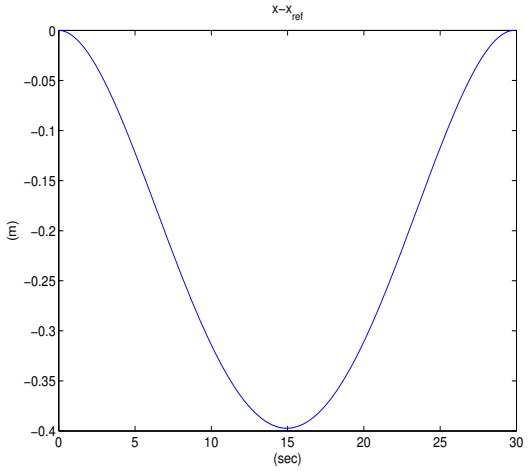

Figure 3.

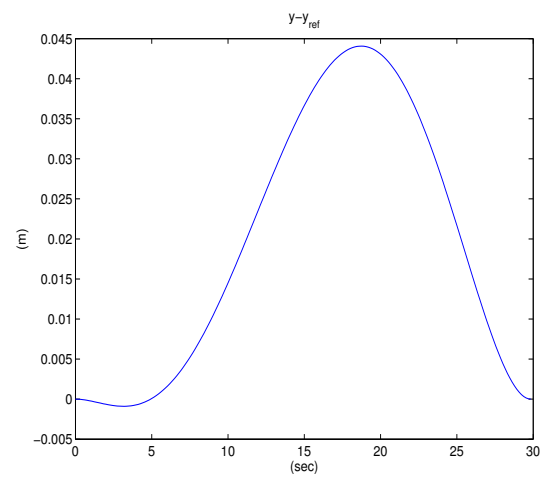

Figure 4 .

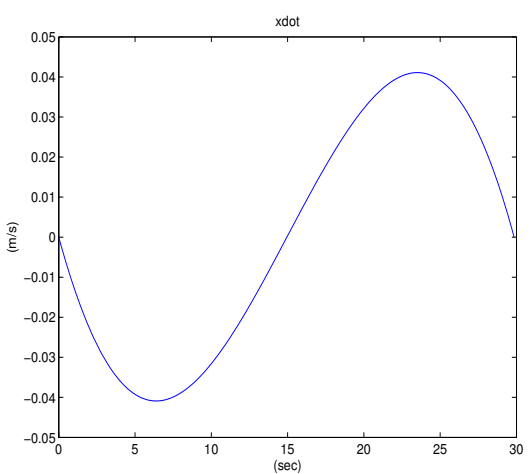

Figure 6.

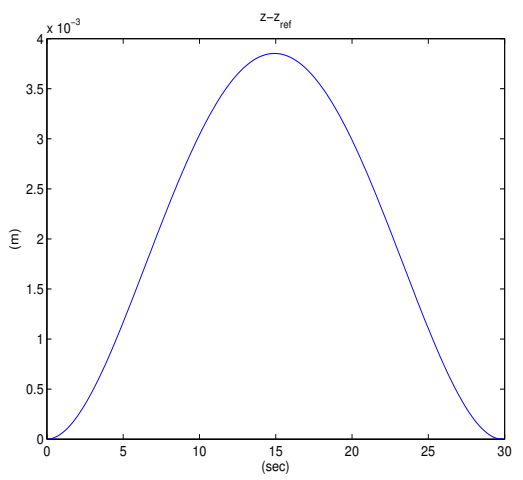

Figure 5.

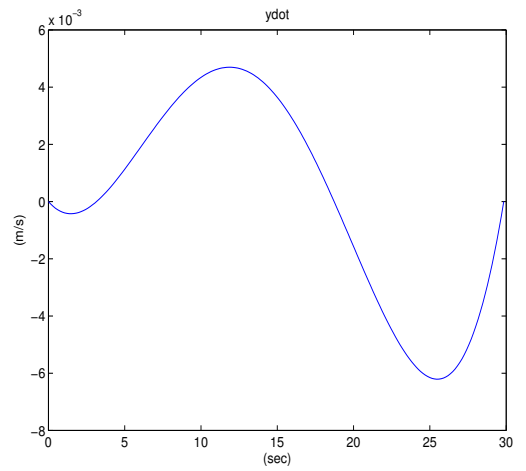

Figure 7. 


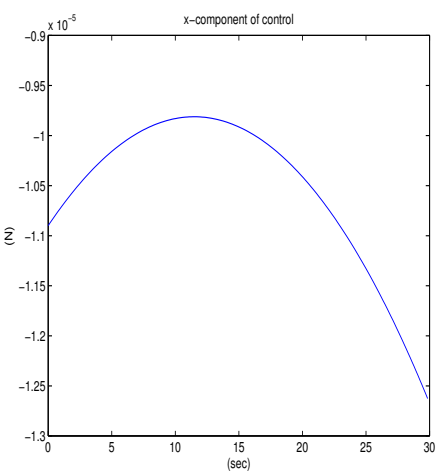

Figure 8.

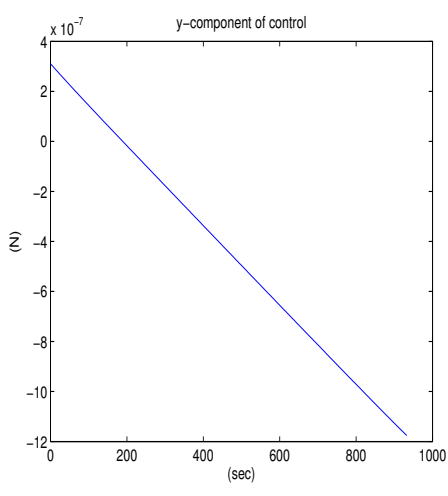

Figure 9.

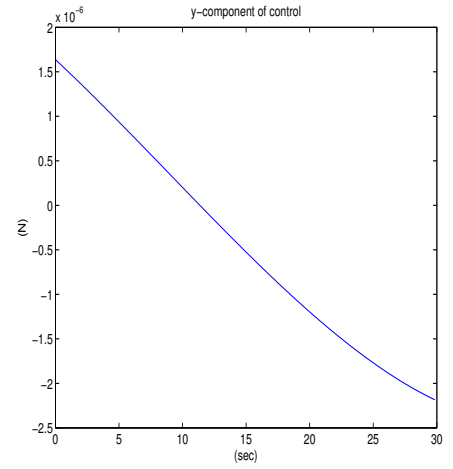

Figure 10 